\title{
Timed Up and Go Test can predict recurrent falls: a longitudinal study of the community-dwelling elderly in China
}

This article was published in the following Dove Press journal:

Clinical Interventions in Aging

\author{
Li Kang ${ }^{1,2}$ \\ Peipei Han ${ }^{2}$ \\ Jiazhong Wang ${ }^{2}$ \\ Yixuan $\mathrm{Ma}^{2}$ \\ Liye Jia ${ }^{2}$ \\ Liyuan $\mathrm{Fu}^{2}$ \\ Hairui $\mathrm{Yu}^{2}$ \\ Xiaoyu Chen ${ }^{2}$ \\ Kaijun $\mathrm{Niu}^{3,4}$ \\ Qi Guo ${ }^{1,2}$ \\ 'Department of Rehabilitation \\ Medicine, Tianjin Economic- \\ Technological Development Area \\ International Cardiovascular \\ Hospital, Cardiovascular Clinical \\ College of Tianjin Medical University, \\ 2Department of Rehabilitation \\ Medicine, ${ }^{3}$ Nutritional Epidemiology \\ Institute, ${ }^{4}$ School of Public Health, \\ Tianjin Medical University, \\ Tianjin, China
}

Correspondence: Qi Guo

Department of Rehabilitation Medicine, TEDA International Cardiovascular Hospital, Cardiovascular Clinical College of Tianjin Medical University, 6I, Third Avenue, TEDA, Tianjin 300457, China

Tel +862283336977

Fax +862283336977

Email guoqijp@gmail.com
Purpose: Falling is a major health problem in community-dwelling elderly individuals. The aim of the present study was to conduct a prospective investigation to evaluate the accuracy of the Timed Up and Go Test (TUGT), 4-meter walking test, and grip strength test to screen for the risk of falls and to determine a cutoff point to be used clinically.

Patients and methods: This was a prospective study that included 541 participants. The fall data were obtained via face-to-face interview, and the date, site, and circumstances of any falls were recorded. TUGTs were recorded as part of a comprehensive geriatric assessment. We collected the same data at baseline and after follow-up via comprehensive geriatric assessment.

Results: The incidence of falls of our study subjects was $20.8 \%$. The recurrent-fall group had a fall rate of $6.8 \%$ during the follow-up year. The standard area under the curve (AUC) of our screening tool was $>0.70$, and hence our tool can be used for clinical purposes. After adjusting for age and gender, the AUC of TUGT became 0.642 , so it cannot be used as a predictive tool for measuring any types of falls. However, when recurrent falls were adjusted for age and gender, the TUGT's AUC improved to 0.733 and a score of 15.96 seconds is used as a cut-point to screen recurrent falls in community-dwelling elderly Chinese individuals.

Conclusion: Future falls were best predicted by TUGT in recurrent fallers at baseline. A score of 15.96 seconds is used as a cut-point to screen recurrent falls in community-dwelling elderly Chinese individuals.

Keywords: community dwelling, elderly, fall, TUGT

\section{Introduction}

Falls are a major problem for elderly people. Approximately $30 \%$ of the population aged $\geq 65$ years fall each year and this percentage rises to $50 \%$ in people $>80$ years old. ${ }^{1}$ Currently, in China more than $14.3 \%$ of the population is $\geq 60$ years and is expected to increase to $34 \%$ in $2050 .^{2}$ As age increases, muscle mass and strength gradually decline, leading to diminished physical performance, ${ }^{3}$ which leads to a high risk of falls and subsequent fractures, loss of independence, and increased morbidity and mortality rates. ${ }^{4,5}$

Gait instability has been identified as a risk factor for falls and the majority of screening programs to identify those at risk of falls comprise an assessment of gait and balance. ${ }^{6}$ Recently, the Timed Up and Go Test (TUGT) has been recommended to assess gait and balance and it has been validated as an appropriate method for evaluating elderly individuals' risk of falling. ${ }^{7}$ However, the literature on its predictive value and diagnostic accuracy for identifying future falls is not straightforward. One systematic 
review showed that although retrospective studies found that the TUGT time performance is associated with a past history of falls, its predictive ability for future falls remains limited. ${ }^{8}$ Additionally, while there is currently no uniform cutoff point for elderly Chinese individuals, another systematic review demonstrated that the diagnostic accuracy of the TUGT is limited to the widely used cutoff point of $\geq 13.5$ seconds, which should not be used clinically to identify communitydwelling adults at a high risk for falls. ${ }^{9}$

Although there have been large-scale clinical trials demonstrating the effectiveness of the TUGT for assessing risk of falls, data on the TUGT's value in the Chinese population are sparse. The results of the TUGT can vary due to race ${ }^{10}$ and population characteristics, eg, community-dwelling versus institutionalized elderly women. ${ }^{11}$ Currently, there are no recommended cutoff points for different Chinese community-dwelling adults. ${ }^{12}$ At present, more than $70 \%$ of the elderly Chinese individuals live in suburban areas. ${ }^{13}$ The current study population is a part of this group, most of whom are farmers (ie, working in the fields) who have not completed a high level of education and have less health care access. Therefore, the aim of the present study was to conduct a prospective investigation to evaluate the accuracy of TUGT, 4-meter walking test, and grip strength test to screen for the risk of falls and to determine a cutoff point to be used clinically among community-dwelling elderly Chinese individuals, specifically in suburban-dwelling elderly populations.

\section{Patients and methods}

\section{Participants}

Our study population comprised residents of the township central hospital of Chadian, in the suburban Hangu of Tianjin, China. They were aged $\geq 60$ years and had joined the China's national free physical examination programs. The participants were fully informed of the nature of research and signed an informed consent to participate. Subjects participated from March 2013 at baseline and from March 2014 at follow-up. All subjects were invited to participate in a comprehensive geriatric assessment. A total of 669 subjects were included in the follow-up group. The exclusion criteria were as follows: 1) inability to perform the basic activities of daily living and thus could not complete performance-based assessments; 2) visual impairments (ie, glaucoma, cataracts, and myopia and presbyopia that were not adequately corrected) that interfered with their daily activities; and 3) current use of drugs (psychotropic drugs, cardiovascular drugs, hypoglycemic agents, non-steroidal anti-inflammatory drugs, analgesics, dopaminergic drugs, Parkinson's disease drugs, or more than four kinds of complex drugs). During the 1-year follow-up, there were missing data on 125 people, one person passed away, and two were unable to complete the assessment. A total of 541 people (age range: 60-86 years) completed the follow-up. This research was approved by the Ethics Committee at Tianjin Medical University.

\section{Comprehensive geriatric assessment}

The comprehensive assessment included a questionnaire, physical performance tests, and a grip strength measurement.

Demographic, behavior performance, and dates of physical illness were obtained via face-to-face interview. ${ }^{14}$ Demographic variables, including age, gender, living status (alone or with another person), and educational level were assessed. A history of physical illness was obtained on the basis of participants' responses (yes or no) to questions about hypertension, hyperlipidemia, stroke, coronary heart disease, and kidney disease, including physician diagnosis, as well as about taking corresponding medication or other treatment now or in the past. Information on smoking ("not currently smoking" and "currently smoking") and drinking ("not currently drinking" and "currently drinking") statuses was also obtained from the questionnaire. Height and weight were recorded using a standard protocol. Body mass index was calculated as weight in kilograms divided by height in meters squared. The Geriatric Depression Scale was used to evaluate depressive symptoms, where participants with a score of $\geq 11$ were considered to have depressive symptoms. ${ }^{15}$ Physical activity level was measured by the average number of hours per day spent in leisure, household, and occupational physical activities over the previous 7-day period, using the short form of the International Physical Activity Questionnaire (IPAQ), in the Chinese language. ${ }^{16}$ Responses were converted to metabolic equivalent task minutes per week (MET-min/week) according to the IPAQ scoring protocol: total minutes over the previous 7 days spent on vigorous activity, moderate-intensity activity, and walking were multiplied by $8.0,4.0$, and 3.3, respectively, to create MET scores for each activity level. MET scores across the three subcategories were summed to indicate overall physical activity. ${ }^{17}$ Gait function was assessed with the 4-meter walk tests. Participants were instructed to stand with both feet touching the starting line and to begin walking at their usual pace after a verbal command. The time between the activation of the first and second photocells was recorded. The average time of two trials was used to compute walking speed. The participants were allowed to use a gait-assistance device. ${ }^{18}$ 
Grip strength $(\mathrm{kg})$ was used as a measure of muscle strength and was quantified using a handheld dynamometer (GRIP-D; Takei Ltd, Niigata, Japan). Participants were asked to exert their maximum effort twice using their dominant hand and the average grip strength used was recorded. ${ }^{19}$

\section{TUGT}

The TUGT assesses the number of seconds needed for an individual to stand up from a chair, walk 3 meter at their usual pace past a line on the floor, turn around, walk back to the chair, and sit down again with the back against the chair. ${ }^{20}$

\section{Falls}

A fall is defined as an event that results in a person coming to rest unintentionally on the ground or other lower level, and the fall not being caused by a violent blow, loss of consciousness, sudden onset paralysis, or epileptic seizure. ${ }^{21}$ The fall data were obtained via face-to-face questioning, and the date, site, and circumstances of any falls at the baseline and after a year of follow-up were recorded. The study population was divided into three groups based on whether they fell zero, one, or two or more times during the 1-year follow-up (no-fall group, one-fall group, and recurrent-fall group, respectively).

\section{Statistical analyses}

Differences between variables were examined by one-way ANOVAs with a Bonferroni correction (continuous variables) or by chi-squared test (categorical variables). Continuous variables were expressed as mean $\pm \mathrm{SD}$ and categorical variables as an absolute number and percentage $(\%)$ of the total. Cox regression analyses were used to examine the relationships between adjusted variables and falls. This analysis was conducted as a subgroup analysis that was stratified according to the times of falling (any and recurrent). Predictive values of the absolute cutoffs of baseline measures of the TUGT, 4-meter walking test, and grip strength test for one or more future falls and recurrent $(>1)$ falls were calculated as the areas under the curve (AUCs) of the receiver operating characteristic (ROC) curves. ROC curves were constructed to analyze sensitivity and specificity, and Bootstrap was used to calculate the AUC confidence intervals. The sensitivity of a test describes how well it correctly identifies subjects with a condition of interest, whereas specificity indicates the frequency that the test is negative in the absence of a condition of interest. ${ }^{22}$ We use logical regression to build models for fall risk, with any fallers or recurrent fallers as the dependent variables, included the TUGT, 4-meter walking test, and grip strength as the predictors, age and gender were independent variables in the models. Two-tailed $P$-values $<0.05$ were considered statistically significant. To build a clinical approach to prevent recurrent falls among elderly individuals living in the community, two cutoffs were discussed: the cutoff maximizing the Youden index and the cutoff maximizing the sum of the positive predictive value (PPV) and negative predictive value (NPV). All statistical analyses were performed with SPSS V17.0 software package (SPSS Inc, Chicago, IL, USA).

\section{Results}

All the 541 subjects completed the TUGT assessment and other tests at the follow-up of 1 year. A total of 113 people fell; 76 subjects (14\%) belonged to the one-fall group and 37 subjects $(6 \%)$ to the recurrent-fall group.

\section{Characteristic of the study population}

The baseline characteristics of population are reported in Table 1. The mean age of the study group was $67.4 \pm 5.6$ years, where 234 were men and 307 were women. The mean value of falls was $2.5 \pm 0.7$, with minimum value being 2 and maximum value 4 for falls in the recurrent-fall group. The subjects in the recurrent-fall group were significantly older than those in the no-fall and one-fall groups. Regardless of fall group, more women fell than men. Of all the models used, assessment only by TUGT showed a significant difference between the two fall groups. The recurrent-fall group used a walking aid significantly more. Moreover, among the three groups, diabetes or stroke patients were more likely to fall than those who had not been diagnosed with diabetes or stroke, while other variables showed no significant differences among the groups. Only the TUGT results showed a significant difference in the one-fall group and recurrent-fall group.

\section{The model for factors related to fall}

Table 2 shows the hazards ratio (HR) and 95\% confidence interval $(95 \% \mathrm{CI})$ of the TUGT, 4-meter walking test, and grip strength test for falls during the follow-up period for the any-fall and recurrent-fall groups. In the any-fall group, a significant association between grip strength and falls was observed (HR: 0.968, 95\% CI: 0.945-0.991); moreover, the 4-meter walking test and falls were significantly associated. However, the TUGT, the 4-meter walking test, or grip strength test after adjusting age and gender, only the TUGT (HR: 1.070, 95\% CI: 1.003-1.142) had a positive association with fall. TUGT showed an association between the recurrent-fall group and falls (HR: 1.160, 95\% CI: 1.061-1.268). 
Table I The baseline's characteristics of study participants (no-fall group, one-fall group, recurrent-fall group) during the follow-up period

\begin{tabular}{|c|c|c|c|c|c|}
\hline \multirow[t]{2}{*}{ Variable } & \multirow{2}{*}{$\begin{array}{l}\text { Total } \\
\mathrm{n}=\mathbf{5 4}\end{array}$} & \multirow{2}{*}{$\frac{\text { No-fall group }}{n=428}$} & \multirow{2}{*}{$\frac{\text { One-fall group }}{n=76}$} & \multirow{2}{*}{$\frac{\text { Recurrent-falls group }}{n=37}$} & \multirow[t]{2}{*}{$P$-value } \\
\hline & & & & & \\
\hline \multicolumn{6}{|l|}{ Demographics } \\
\hline Age (years) & $67.4 \pm 5.6$ & $66.9 \pm 5.5$ & $68.3 \pm 6.1^{*}$ & $70.6 \pm 5.2 *$ & $<0.001$ \\
\hline Gender (male/female) & $234 / 307$ & $198 / 230$ & $26 / 50 *$ & $10 / 27^{*}$ & 0.022 \\
\hline Widowed (\%) & 16.1 & 14.6 & 20.1 & 19.4 & 0.418 \\
\hline Walking aid (\%) & 0.4 & 0.0 & $1.3^{*}$ & $2.8^{*}$ & 0.001 \\
\hline Living alone (\%) & 11.1 & 11.3 & 7.8 & 13.9 & 0.580 \\
\hline Fall history (\%) & 9.3 & 6.8 & $|7 .|^{*}$ & $22.2 *$ & $<0.001$ \\
\hline Illiterate (\%) & 43.3 & 43.9 & 40.8 & 41.7 & 0.862 \\
\hline Drinking (\%) & 37.2 & 37.1 & 39.5 & 33.3 & 0.818 \\
\hline Smoking (\%) & 51.9 & 50.9 & 52.6 & 61.1 & 0.497 \\
\hline \multicolumn{6}{|l|}{ Objective parameters } \\
\hline Grip/weight (kg/kg) & $0.37 \pm 0.11$ & $0.38 \pm 0.11$ & $0.35 \pm 0.10 *$ & $0.34 \pm 0.12 *$ & 0.011 \\
\hline $\begin{array}{l}\text { 4-meter walking } \\
\text { test }(\mathrm{m} / \mathrm{s})\end{array}$ & $\mathrm{I} .0 \mathrm{I} \pm 0.2 \mathrm{I}$ & $1.02 \pm 0.21$ & $0.98 \pm 0.22 *$ & $0.90 \pm 0.20 *$ & 0.003 \\
\hline TUGT (s) & $10.3 \pm 2.5$ & $10.1 \pm 2.3$ & $10.6 \pm 2.4 *$ & $12.5 \pm 4 . I^{*, * *}$ & $<0.001$ \\
\hline BMI $\left(\mathrm{kg} / \mathrm{m}^{2}\right)$ & $25.7 \pm 3.5$ & $25.7 \pm 3.5$ & $25.7 \pm 3.3$ & $25.4 \pm 3.6$ & 0.889 \\
\hline IPAQ (MET-min/week) & $3,909.9 \pm 5,910.2$ & $4,130.3 \pm 6,346.9$ & $3,152.9 \pm 3,824.9$ & $2,900.9 \pm 3,542.9$ & 0.236 \\
\hline $\begin{array}{l}\text { Depression } \\
\quad \text { GDS } \geq \text { II (\%) }\end{array}$ & 9.5 & 7.5 & $11.8^{*}$ & $27.8^{*}$ & $<0.001$ \\
\hline \multicolumn{6}{|l|}{ Diseases } \\
\hline Diabetes (\%) & 9.3 & 7.7 & $13.2^{*}$ & $19.4 *$ & 0.031 \\
\hline Hypertension (\%) & 45.2 & 46.0 & 44.7 & 36.1 & 0.517 \\
\hline Hyperlipidemia (\%) & 6.7 & 6.1 & 10.5 & 5.6 & 0.350 \\
\hline Heart disease (\%) & 23.2 & 22.1 & 26.3 & 30.6 & 0.404 \\
\hline Peptic ulcer (\%) & 6.3 & 6.1 & 5.3 & II.I & 0.455 \\
\hline Stroke (\%) & 7.2 & 6.1 & $14.5^{*}$ & $5.6^{*}$ & 0.032 \\
\hline Gout (\%) & 2.2 & 2.3 & 1.3 & 2.8 & 0.832 \\
\hline Cancer (\%) & 1.3 & 1.4 & 1.3 & 0.0 & 0.774 \\
\hline Kidney disease (\%) & 2.8 & 2.8 & 2.6 & 2.8 & 0.996 \\
\hline Hepatic disease (\%) & 1.3 & 1.4 & 0.0 & 2.8 & 0.438 \\
\hline Biliary tract disease (\%) & 4.8 & 4.5 & 5.3 & 8.3 & 0.574 \\
\hline Thyroid disease (\%) & 1.9 & 2.1 & 1.3 & 0.0 & 0.620 \\
\hline Osteoarthritis (\%) & 25.8 & 25.4 & 22.4 & 38.9 & 0.155 \\
\hline Anemia (\%) & 1.3 & 1.6 & 0.0 & 0.0 & 0.394 \\
\hline
\end{tabular}

Notes: Data are presented as means \pm SD for age, body mass index (BMI), grip strength, Timed Up and Go Test (TUGT), 4-meter walking test, and International Physical Activity Questionnaire (IPAQ). All other variables are in percentages; $* P<0.05$ versus no-fall group; $* * P<0.05$ versus one-fall group; $P$-value of the ANOVA among the three groups in the table.

Abbreviations: GDS, Geriatric Depression Scale; MET-min/week, metabolic equivalent task minutes per week.

Table 2 Hazard ratio (HR) and 95\% confidence interval of baseline TUGT, 4-meter walking test, and grip strength for any-fall group and recurrent-fall group during the follow-up period

\begin{tabular}{|c|c|c|c|c|}
\hline \multirow[t]{2}{*}{ Model } & \multicolumn{2}{|l|}{ Any-fall group } & \multicolumn{2}{|l|}{ Recurrent-fall group } \\
\hline & Non-fallers $(n=428)$ & Fallers $(n=113)$ & Non-fallers $(n=504)$ & Fallers $(n=37)$ \\
\hline \multicolumn{5}{|l|}{ Grip strength } \\
\hline No adjustment & 1.000 (reference) & $0.968(0.945-0.991)^{*}$ & 1.000 (reference) & $0.957(0.917-0.999) *$ \\
\hline Adjusted for age and gender & 1.000 (reference) & $1.000(1.000-1.000)$ & 1.000 (reference) & $1.000(1.000-1.001)$ \\
\hline \multicolumn{5}{|l|}{ 4-meter walking test } \\
\hline No adjustment & 1.000 (reference) & $0.272(0.100-0.739)^{*}$ & 1.000 (reference) & $0.037(0.006-0.214)^{*}$ \\
\hline Adjusted for age and gender & 1.000 (reference) & $1.005(0.999-1.010)$ & 1.000 (reference) & $1.004(0.994-1.013)$ \\
\hline \multicolumn{5}{|l|}{ TUGT } \\
\hline No adjustment & 1.000 (reference) & $1.098(1.039-1.161)^{*}$ & 1.000 (reference) & I.I8I (I.094-I.275)* \\
\hline Adjusted for age and gender & I.000 (reference) & $1.070(1.003-1.142)^{*}$ & I.000 (reference) & $1.160(1.06 \mathrm{I}-1.268)^{*}$ \\
\hline
\end{tabular}

Note: $* P<0.05$ versus same group referent values.

Abbreviation: TUGT, Timed UP and Go Test. 
Table 3 Diagnostic value of baseline TUGT, 4-meter walking test, and grip strength measures for any fall and recurrent fall

\begin{tabular}{|c|c|c|c|c|}
\hline \multirow[t]{3}{*}{ Models } & \multicolumn{2}{|c|}{ Prediction of any falls } & \multicolumn{2}{|c|}{ Prediction of recurrent falls } \\
\hline & \multicolumn{2}{|l|}{$n=|| 3 / 54 \mid$} & \multicolumn{2}{|l|}{$n=37 / 541$} \\
\hline & AUC $^{a}$ value & $95 \% \mathrm{Cl}$ & AUC $^{a}$ value & $95 \% \mathrm{Cl}$ \\
\hline \multicolumn{5}{|l|}{ Grip strength } \\
\hline Grip strength ${ }^{\mathrm{b}}$ & 0.512 & $0.449-0.574$ & 0.548 & $0.429-0.666$ \\
\hline Grip strength ${ }^{c}$ & 0.602 & $0.542-0.663$ & 0.612 & $0.511-0.712$ \\
\hline \multicolumn{5}{|l|}{ 4-meter walking test } \\
\hline 4-meter walking test ${ }^{\mathrm{b}}$ & 0.563 & $0.504-0.622$ & 0.542 & $0.445-0.639$ \\
\hline 4-meter walking test ${ }^{c}$ & 0.586 & $0.526-0.647$ & 0.680 & $0.593-0.768$ \\
\hline \multicolumn{5}{|l|}{ TUGT } \\
\hline TUGT $^{\mathrm{b}}$ & 0.607 & $0.549-0.665$ & 0.688 & $0.602-0.773$ \\
\hline TUGTc & 0.642 & $0.584-0.700$ & 0.733 & $0.645-0.821$ \\
\hline
\end{tabular}

Notes: ${ }^{2}$ Areas under the curve (AUCs) of the receiver operating characteristic (ROC) curves for grip strength, 4-meter walking test, and TUGT for fallers versus non-fallers and recurrent fallers versus non-fallers; ${ }^{b}$ no adjustment; cadjusted for gender and age.

Abbreviations: $\mathrm{Cl}$, confidence interval; TUGT, Timed Up and Go Test.

The results obtained by TUGT, 4-meter walking test, and grip strength test were analyzed by logistic regression models and the results showed low predictive values for any-fall and recurrent-fall groups (Table 3). The TUGT, the 4-meter walking test, or grip strength test after adjusting age and gender, the predictive value was not high for the any-fall group. Table 2 shows the results obtained by all the three models with and without adjustment for age and gender; no significant associations were found among the groups by all the models tested $(P>0.05)$. Also, no significant predictive value was obtained by the 4-meter walking test (AUC 0.680) and grip strength test (AUC 0.612 ) for the recurrent-fall group. However, TUGT after adjusted for age and gender showed a slightly better predictive value for recurrent-fall group (AUC 0.733) (Figure 1).

\section{Further analysis of TUGT}

Based on the Youden index, it was found that the most predictive cutoff point of TUGT for elderly individuals who recurrently fell was 10.15 seconds, with $67.5 \%$ sensitivity and $56.3 \%$ specificity. Using 10.15 seconds as the cutoff time for the TUGT, the PPV and NPV were calculated, which were $10.2 \%$ and $95.9 \%$, respectively. With a relative low cutoff point, the sensitivity was moderate and the specificity was low; with an increase in the cutoff point, the sensitivity decreased, whereas the specificity increased. Based on the assessment of the PPV and NPV, the best cutoff point could be 15.96 seconds (13.9\% sensitivity and $98.4 \%$ specificity; PPV $70.0 \%$ and NPV $81.3 \%$ ).

The TUGT as a continuous predictor had an HR of 1.160 (95\% CI 1.061-1.268, $P<0.001$ ). Table 2 shows that the value of TUGT increases by one unit ( 1 second), there was a $16 \%$ increased the risk of recurrent falls.

\section{Discussion}

This study showed that the TUGT as a simple, single-item tool has low predictive value for any falls and moderate predictive values for recurrent falls. The TUGT after adjusting the age and gender, was slightly better at predicting future recurrent falls in community-dwelling elderly individuals. Moreover, the cutoff value of 15.96 seconds seems to be

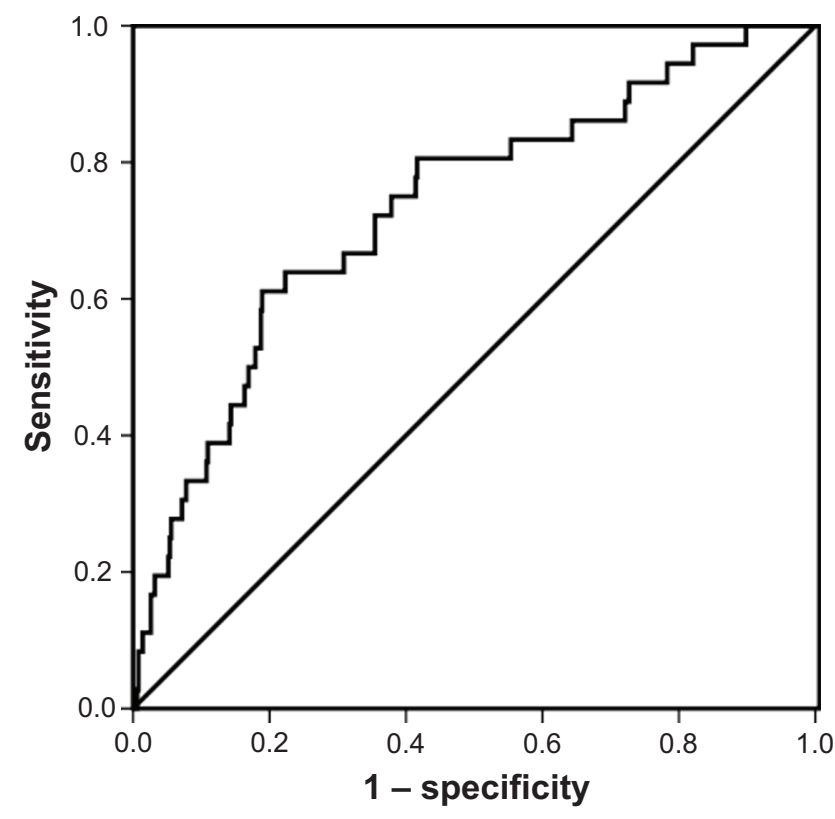

Figure I ROC curve of TUGT as predictor of recurrent falls among elderly individuals (area under ROC curve $=0.733$ [95\% Cl: $0.645-0.82$ I]).

Abbreviations: ROC, receiver operating characteristic; TUGT, Timed Up and Go Test; $\mathrm{Cl}$, confidence interval. 
a better predictive value to screen for recurrent falls in the current study population of elderly individuals.

We included age and gender as predictors in our model because our group's previous studies have found that age might be an important factor in affecting balance among women. ${ }^{23}$ Also a systematic review has found that standardization of testing conditions along with control of significant potential confounders (age and gender) would provide better information about the predictive value of TUGT to measure future falls in older adults. ${ }^{8}$ Among the three groups, recurrent fallers were most likely to be female and the oldest of the participants (Table 1). Our study found that advanced age and female gender were risk factors for falls, which is in accordance with most of the studies carried out in Western and other Chinese countries. ${ }^{24,25}$ Therefore, we selected risks factors for falls that previous research had identified: age and gender.

Our study used the standard AUC $>0.70$, which is used in clinical practice..$^{26,27}$ Therefore, TUGT can be an effective, simple, and practical tool to predict future recurrent falls in elderly people. This study also did confirm the earlier findings that TUGT is better in predicting recurrent falls, and adding age and gender to the model increases the predictive ability of TUGT. ${ }^{10}$ However, there is controversy over the predictive value of TUGT. A previous research found that the predictive values obtained using a more reliable follow-up assessment of falls was not the same as those values obtained using TUGT. ${ }^{28}$ This discrepancy is possibly related to the characteristics of the different study populations. Our study population comprised younger individuals (67.2 \pm 5.6$)$, whereas the aforementioned study comprised elderly individuals (84.4 \pm 5.3$)$. So, TUGT may be more suitable for our population.

We used two cutoff points: one based on the Youden index (10.15) and the other based on the assessment of PPV and NPV (15.96). However, a recent systematic review and meta-analysis indicated that the TUGT is more useful at ruling out the possibility of falls rather than positively identifying individuals classified as high risk. ${ }^{9}$ Previous studies ${ }^{26,29}$ also suggested that the appropriate cutoff must be chosen according to the relative costs (not necessarily financial) of the screening test, which is related to the number of false positives and false negatives and to the prevention strategy that follows after a positive test. If a risk tool is used to select elderly individuals to participate in preventive programs, a cutoff ensuring a relatively high sensitivity is needed so that most of the participants who could become future fallers are identified. In contrast, when the risk tool is used to select subjects for further diagnostic testing in specialized medical settings, a high specificity is needed to ensure that non-fallers will not receive a tiring and costly examination conducted by other medical specialists. We hope the TUGT will be used in primary health care and in hospitals as a screening test for the risk of falls and will allow clinicians to make a definite decision about when an intervention is necessary. Thus, the cutoff value of 15.96 seconds suggested based on the assessment of PPV and the NPV would be the most appropriate.

The findings (Table 2) showed that a higher 4-meter walking test at baseline resulted in a lower risk of falling. These results are in accordance with those of the previous studies. ${ }^{30,31}$ The present study also found that grip strength may be associated with falls, and previous studies showed that reduced muscle strength plays an important role in falls in older adults. ${ }^{32,33}$ However, in the full model, the 4-meter walking test and grip strength test are weakly correlated to falls. The fact that 4-meter walking test or grip strength and falls are independent of each other in our study may be explained by previous studies, ${ }^{34,35}$ which found a nonlinear relationship between 4-meter walking test or grip strength and falls. Given all these, in our population, one must consider other risk factors into account when using 4-meter walking test or grip strength test to study falls.

\section{Limitations}

There were some limitations. First, we could not assess the complete population of elderly individuals; our participants were relatively healthy, community-dwelling older adults who volunteered to participate in health checkups. Thus, the population studied may not be comprehensive enough, so our findings may be weakened by selection bias. Second, our study's follow-up was short; as a result, the change in elderly health status over the short observation time is small. Third, although the cutoff value of 15.96 seconds obtained based on the assessment of PPV and NPV would be the most appropriate, the cutoff point of TUGT cannot be the same for older adults of 60 years and 80 years. Therefore, in future research, age should be stratified to obtain an optimum cutoff point for each age group. Fourth, there are other parameters of interest in the TUGT (eg, number of steps) that we did not measure in this study. Although the number of steps does not affect the measurement of TUGT, in future research, we will take into account all the parameters of TUGT. Finally, we used only TUGT to predict falls. We are designing a longitudinal study to be carried out in future, with an aim to develop a comprehensive clinical intervention tool (including TUGT 
combined with other risk factors) that yields relatively higher predictive values when assessing for risk of falls in elderly Chinese individuals.

\section{Conclusion}

In summary, TUGT could predict falls. The cutoff value of 15.96 seconds seems to be a better predictive value to screen recurrent falls in community-dwelling elderly Chinese individuals.

\section{Acknowledgment}

The authors thank Peipei Han from the Department of Rehabilitation Medicine, Tianjin Medical University, for processing data and writing documents. This work was supported by the National Natural Science Foundation of China (81372118) and Tianjin Municipal Science and Technology Commission (grant number 16ZXMJSY00070).

\section{Disclosure}

The authors report no conflicts of interest in this work.

\section{References}

1. Soriano TA, DeCherrie LV, Thomas DC. Falls in the communitydwelling older adult: a review for primary-care providers. Clin Interv Aging. 2007;2(4):545-554.

2. Shi J, Zhou BY, Tao YK, et al. Incidence and associated factors for single and recurrent falls among the elderly in an urban community of Beijing. Biomed Environ Sci. 2014;27(12):939-949.

3. Shin H, Panton LB, Dutton GR, Ilich JZ. Relationship of physical performance with body composition and bone mineral density in individuals over 60 years of age: a systematic review. J Aging Res. 2011; 2011:191896.

4. Stenholm S, Harkanen T, Sainio P, Heliovaara M, Koskinen S. Longterm changes in handgrip strength in men and women - accounting the effect of right censoring due to death. J Gerontol A Biol Sci Med Sci. 2012;67(10):1068-1074.

5. den Ouden ME, Schuurmans MJ, Arts IE, van der Schouw YT. Physical performance characteristics related to disability in older persons: a systematic review. Maturitas. 2011;69(3):208-219.

6. Ganz DA, Bao Y, Shekelle PG, Rubenstein LZ. Will my patient fall? JAMA. 2007;297(1):77-86.

7. Panel on Prevention of Falls in Older Persons American Geriatrics Society, British Geriatrics Society. Summary of the Updated American Geriatrics Society/British Geriatrics Society clinical practice guideline for prevention of falls in older persons. J Am Geriatr Soc. 2011;59(1): $148-157$.

8. Beauchet O, Fantino B, Allali G, Muir SW, Montero-Odasso M, Annweiler C. Timed Up and Go test and risk of falls in older adults: a systematic review. J Nutr Health Aging. 2011;15(10):933-938.

9. Barry E, Galvin R, Keogh C, Horgan F, Fahey T. Is the Timed Up and Go test a useful predictor of risk of falls in community dwelling older adults: a systematic review and meta-analysis. BMC Geriatr. 2014;14:14.

10. Alexandre TS, Meira DM, Rico NC, Mizuta SK. Accuracy of Timed Up and Go Test for screening risk of falls among community-dwelling elderly. Rev Bras Fisioter. 2012;16(5):381-388.

11. Bischoff HA, Stahelin HB, Monsch AU, et al. Identifying a cut-off point for normal mobility: a comparison of the timed 'up and go' test in community-dwelling and institutionalised elderly women. Age Ageing. 2003;32(3):315-320.
12. Schoene D, Wu SM, Mikolaizak AS, et al. Discriminative ability and predictive validity of the timed up and go test in identifying older people who fall: systematic review and meta-analysis. J Am Geriatr Soc. 2013; 61(2):202-208.

13. National Bureau of Statistics of China. Statistical Communiqué of the People's Republic of China on the 2012 National Economy and Social Development. China Statistics Press. 2013. Available from: http:// www.stats.gov.cn/english/PressRelease/201402/t20140224_515103. html. Accessed September 1, 2017.

14. Zhang W, Shen S, Wang W, et al. Poor lower extremity function was associated with pre-diabetes and diabetes in older chinese people. PLoS One. 2014;9(12):e115883.

15. Jongenelis K, Pot AM, Eisses AM, et al. Diagnostic accuracy of the original 30-item and shortened versions of the Geriatric Depression Scale in nursing home patients. Int J Geriatr Psychiatry. 2005; 20(11):1067-1074.

16. Jiang CQ, Xu L, Lam TH, et al. [Effect of physical activity strength on the diabetes mellitus prevalence in the elderly under the influence of International Physical Activity Questionnaire]. Zhonghua Liu Xing Bing Xue Za Zhi. 2009;30(5):462-465. Chinese.

17. Craig CL, Marshall AL, Sjostrom M, et al. International physical activity questionnaire: 12-country reliability and validity. Medicine Sci Sports Exerc. 2003;35(8):1381-1395.

18. Lauretani F, Russo CR, Bandinelli S, et al. Age-associated changes in skeletal muscles and their effect on mobility: an operational diagnosis of sarcopenia. J Appl Physiol. 2003;95(5):1851-1860.

19. Stenholm S, Sallinen J, Koster A, et al. Association between obesity history and hand grip strength in older adults - exploring the roles of inflammation and insulin resistance as mediating factors. J Gerontol A Biol Sci Med Sci. 2011;66(3):341-348.

20. Podsiadlo D, Richardson S. The timed "Up \& Go": a test of basic functional mobility for frail elderly persons. J Am Geriatr Soc. 1991;39(2): $142-148$.

21. Lamb SE, Jorstad-Stein EC, Hauer K, Becker C; Prevention of Falls Network Europe and Outcomes Consensus Group. Development of a common outcome data set for fall injury prevention trials: the Prevention of Falls Network Europe consensus. J Am Geriatr Soc. 2005;53(9): 1618-1622.

22. Sackett DL. The rational clinical examination. A primer on the precision and accuracy of the clinical examination. JAMA. 1992;267(19): 2638-2644.

23. Shen S, Li J, Guo Q, et al. Body mass index is associated with physical performance in suburb-dwelling older chinese: a cross-sectional study. PLoS One. 2015;10(3):e0119914.

24. Chu LW, Chi I, Chiu AY. Incidence and predictors of falls in the chinese elderly. Ann Acad Med Singapore. 2005;34(1):60-72.

25. Teno J, Kiel DP, Mor V. Multiple stumbles: a risk factor for falls in community-dwelling elderly. A prospective study.J Am Geriatr Soc. 1990; 38(12):1321-1325.

26. Bongue B, Dupre C, Beauchet O, Rossat A, Fantino B, Colvez A. A screening tool with five risk factors was developed for fall-risk prediction in community-dwelling elderly. J Clin Epidemiol. 2011; 64(10):1152-1160.

27. Bongers KT, Schoon Y, Graauwmans MJ, Schers HJ, Melis RJ, Olde Rikkert MG. The predictive value of gait speed and maximum step length for falling in community-dwelling older persons. Age Ageing. 2015;44(2):294-299.

28. Beauchet O, Allali G, Annweiler C, et al. Does change in gait while counting backward predict the occurrence of a first fall in older adults? Gerontology. 2008;54(4):217-223.

29. Pluijm SM, Smit JH, Tromp EA, et al. A risk profile for identifying community-dwelling elderly with a high risk of recurrent falling: results of a 3-year prospective study. Osteoporos Int. 2006;17(3): $417-425$.

30. Stone E, Skubic M, Rantz M, Abbott C, Miller S. Average in-home gait speed: investigation of a new metric for mobility and fall risk assessment of elders. Gait Posture. 2015;41(1):57-62. 
31. Gimmon Y, Barash A, Debi R, et al. Application of the clinical version of the narrow path walking test to identify elderly fallers. Arch Gerontol Geriatr. 2016;63:108-113.

32. Muir SW, Beauchet O, Montero-Odasso M, Annweiler C, Fantino B, Speechley M. Association of executive function impairment, history of falls and physical performance in older adults: a cross-sectional population-based study in eastern France. J Nutr Health Aging. 2013; 17(8):661-665.

33. Granacher U, Gollhofer A, Hortobagyi T, Kressig RW, Muehlbauer T. The importance of trunk muscle strength for balance, functional performance, and fall prevention in seniors: a systematic review. Sports Med. 2013;43(7):627-641.
34. Quach L, Galica AM, Jones RN, et al. The nonlinear relationship between gait speed and falls: the Maintenance of Balance, Independent Living, Intellect, and Zest in the Elderly of Boston Study. J Am Geriatr Soc. 2011;59(6):1069-1073.

35. Pavol MJ, Owings TM, Foley KT, Grabiner MD. Influence of lower extremity strength of healthy older adults on the outcome of an induced trip. J Am Geriatr Soc. 2002;50(2):256-262.

\section{Publish your work in this journal}

Clinical Interventions in Aging is an international, peer-reviewed journal focusing on evidence-based reports on the value or lack thereof of treatments intended to prevent or delay the onset of maladaptive correlates of aging in human beings. This journal is indexed on PubMed Central, MedLine,
CAS, Scopus and the Elsevier Bibliographic databases. The manuscript management system is completely online and includes a very quick and fair peer-review system, which is all easy to use. Visit http://www.dovepress. com/testimonials.php to read real quotes from published authors. 